\title{
Análise de parcimônia de endemismo de cercopídeos neotropicais (Hemiptera, Cercopidae)
}

\author{
Ângela Goldani ${ }^{1}$ \\ Gervásio Silva Carvalho ${ }^{1}$
}

\begin{abstract}
Aвstract. Parsimony analysis of endemicity of Neotropical spittlebugs (Hemiptera, Cercopidae). The spittlebugs have an extent distribution in the American continent. Their diversity may determinate endemism areas based on their occurence in different localities. We have used Parsimony Analysis of Endemicity method, which is an important historic biogeography tool for detecting and establishing the relationship among endemics areas. A data matrix was built up based on the occurence registration for the species by 66 genus in whole localities divided in five degrees quadrats in the Neotropical Region, using 49 OGUs (Operative Geografic Units). The presence of the taxa in the areas was coded 1 and the absence 0 . The data matrix was analysed based on parsimony analysis through the computer program Hennig 86. Nine endemic areas were stipulated (Mexico + Central America, Venezuelan Savana, Guiana + Suriname, Chaco, Trans-andean, Cerrado, Amazon, Pampa and Atlantic Forest) in the first analysis corroborated with ecological and physiographic patterns in each region. The second analysis was made using 48 genera to obtain the relationship among the nine areas stipulated before. In this analysis just one cladogram $(3((1,2)((8,9)(6(7(4,5))))))$ was obtained with 192 steps, consistence index 0.80 and retention index 0.85 .
\end{abstract}

KeYwords. Areas of endemism; distribution; Neotropical Region; parsimony analysis of endemicity; spittlebugs.

\section{INTRODUÇÃO}

Dados biogeográficos referenciados através de províncias zoogeográficas, subprovíncias e áreas de endemismo são essenciais para a análise da distribuição da fauna da Terra e suas causas. Interpretações históricas e ecológicas da biogeografia permitem responder " Por que os táxons estão distribuídos onde ocorrem atualmente?” (PlatnicK \& NELSON 1978). A ocorrência exclusiva de um táxon em uma localidade ou região em particular (táxons endêmicos) é uma das características mais significantes da distribuição biogeográfica (ESPINOSA \& LLORENTE 1993). Toda região teria sua biota própria e exclusiva sem qualquer área de sobreposição com outras regiões. Porém, na prática, isto não ocorre ou, pelo menos, é muito raro.

Áreas de endemismo são, fundamentalmente, entidades históricas e não apenas distribucionais (HAROLD \& MoOI 1994) possuindo as mesmas propriedades ontológicas e genealógicas dos grupos nelas distribuídos (Zunino 2000).

A Região Neotropical é um ponto referencial importante no estudo dos padrões de distribuição dos seres vivos por apresentar uma biodiversidade alta e características climáticas e fisiográficas diversificadas.

Para a aplicação de métodos da biogeografia histórica, algumas considerações sobre o grupo estudado devem ser levadas em conta, tais como filogenia e endemismo. Outro fator importante para o estudo distribucional de um determinado táxon é o conhecimento da sua capacidade e limite de dispersão.

Os Cercopidae (Hemiptera) têm uma distribuição ampla na Região Neotropical, mas com restrições que seguem padrões de cada bioma, podendo então serem determinadas áreas de endemismo para este grupo. Cercopídeos são cigarrinhas saltadoras de tamanho variável, desde alguns milímetros até cerca de $4 \mathrm{~cm}$, sugadoras de seiva de diversas espécies de plantas.

A visão de como a endemicidade das espécies está distribuída em uma região representa um passo fundamental para estudos biogeográficos e até mesmo para a biologia da conservação. As informações obtidas pela análise de áreas de endemismo e suas relações podem ser a chave para determinar prioridades na seleção de áreas para a conservação de diferentes ecossistemas na Região Neotropical.

O método de Análise de Parcimônia de Endemismo (PAE) utilizado foi primeiramente proposto por Rosen (1988) e melhor

\footnotetext{
1. Pontifícia Universidade Católica do Rio Grande do Sul, Faculdade de Biociências, Departamento de Biologia. Avenida Ipiranga 6681, Caixa
} Postal 1429, 90619-900 Porto Alegre-RS, Brasil. Bolsistas do CNPq. Endereços eletrônicos: agoldani@yahoo.com; gervasio@pucrs.br 
desenvolvido em Rosen \& SMITH (1988). É um método análogo a métodos cladísticos utilizados em análises filogenéticas, agrupando localidades (semelhante a táxons em cladística), pelos seus táxons compartilhados (semelhantes a caracteres em cladística) (Myers 1991). A análise de parcimônia é aplicada aos táxons que compartilham diferentes localidades para inferir relacionamento entre as biotas amostradas nessas localidades, produzindo cladogramas de área diretamente das distribuições geográficas. O ponto de partida para a análise é a matriz de dados, obtida com base na ocorrência de táxons no conjunto de áreas amostradas. Na matriz, as unidades operacionais são representadas pelas áreas (Operative Geografic Units - OGUs). A presença do táxon é tomada como o estado derivado (codificado como 1) e a ausência como primitivo (0). Analogamente à sistemática, o grupo externo é concebido hipoteticamente como sendo uma área primitiva, sem a presença de qualquer táxon (codificado como zero para todos) (Rosen 1988).

Morrone (1994) propõe delimitar as áreas de endemismo utilizando, como unidades operacionais geográficas, quadrículas a partir das linhas geográficas latitudinais e longitudinais, que são reunidas pela presença compartilhada dos táxons. Seu método pode ser referenciado como Análise de Parcimônia de Endemismo baseado em quadrículas (CRISCI et al. 2000).

\section{MATERIAL E MÉTODOS}

Os dados distribucionais dos gêneros de cercopídeos neotropicais utilizados nas análises, provêm de catálogo (Metcalf 1961) e das etiquetas de coleta dos espécimens em coleções como BMNH (The Natural History Museum, London,

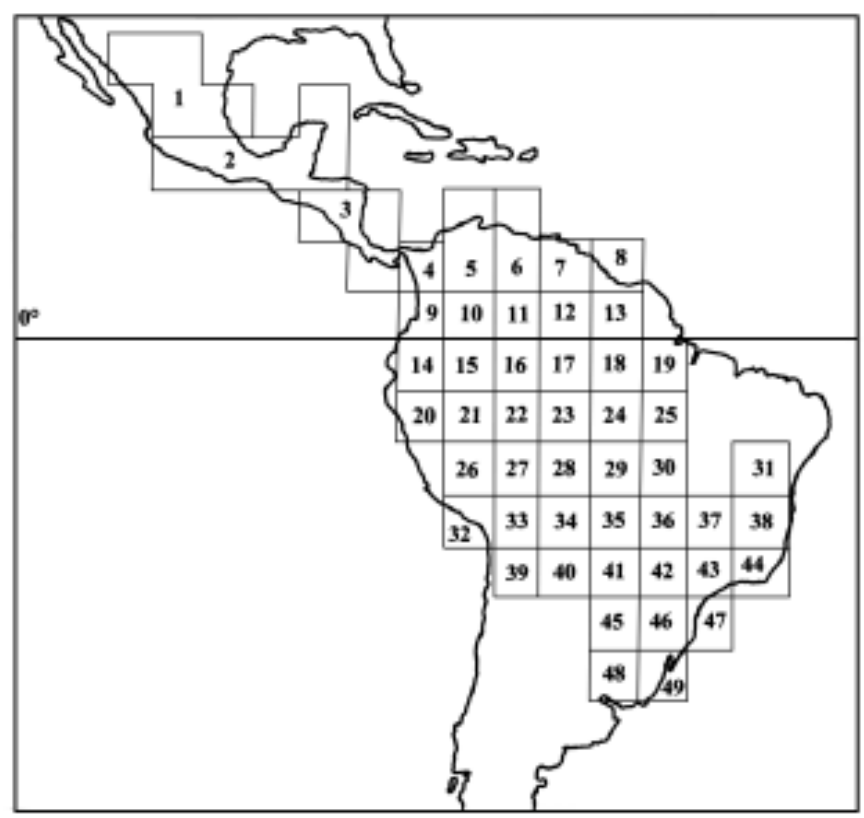

Fig. 1. Mapa da Região Neotropical com as quadrículas utilizadas na $1^{\mathrm{a}}$ análise.
U.K.), DZUP (Departamento de Zoologia, Universidade Federal do Paraná, Curitiba, Brasil) e MCTP (Museu de Ciências e Tecnologia da Pontifícia Universidade Católica do Rio Grande do Sul, Porto Alegre, Brasil).

Para a primeira análise, objetivando determinar áreas de endemismo, foi construída uma matriz na qual as unidades geográficas operacionais constituíram-se de 49 quadrículas (a maioria de $5^{\circ}$ de latitude por $5^{\circ}$ de longitude, Fig. 1) contra dados distribucionais de 66 gêneros de cercopídeos (Tabelas I e II). Posteriormente, em uma segunda análise, determinaramse as relações entre as áreas de endemismo anteriormente identificadas. Para isso, foram utilizados 48 dos 66 gêneros, excluíndo-se aqueles ocorrentes em uma só área (análogo à autapomorfia) (Tabela III).

As análises foram realizadas com auxílio do programa computacional Hennig86 (FARRIs 1988).

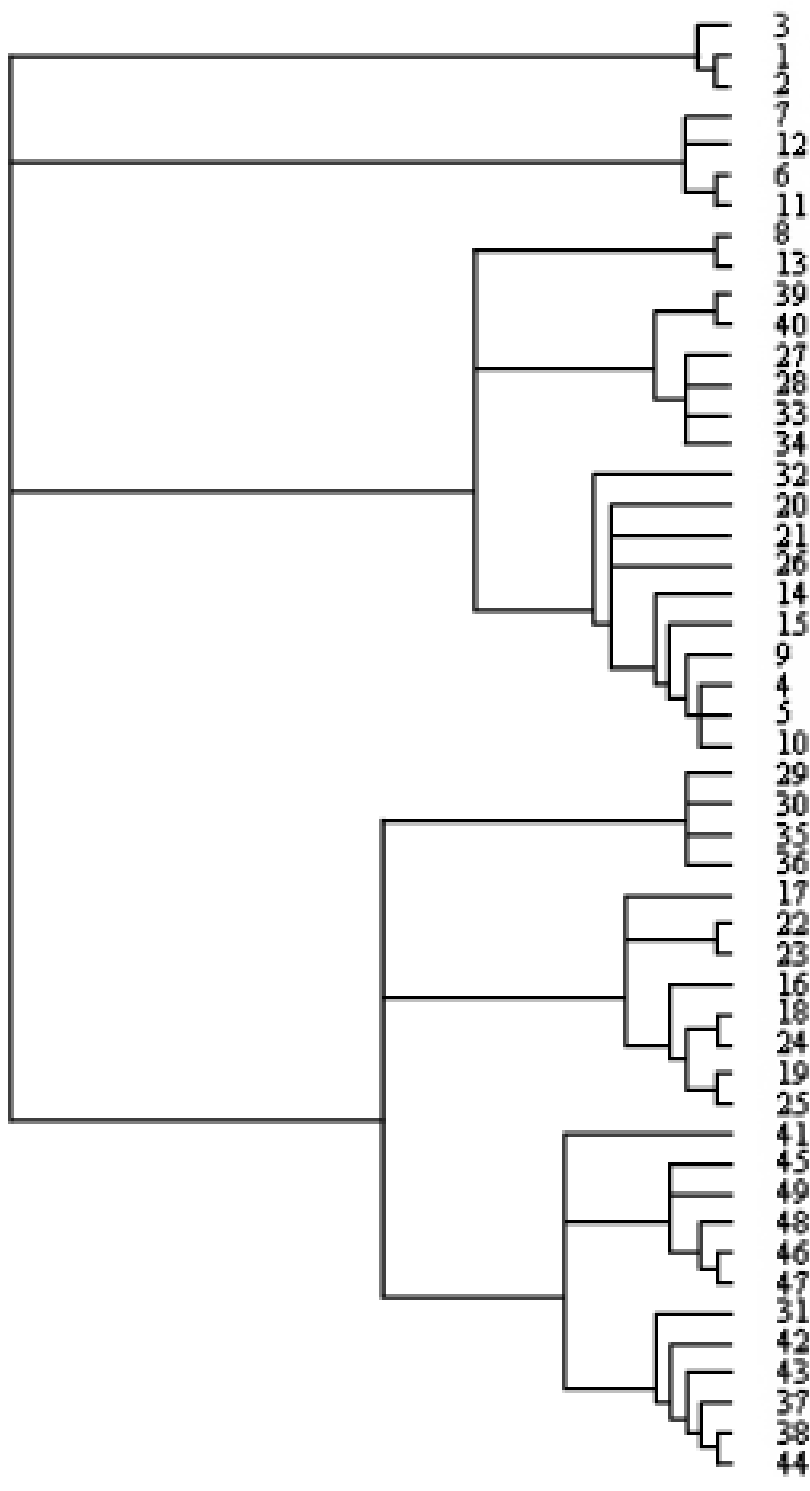

Fig. 2. Cladograma de consenso resultante da $1^{\mathrm{a}}$ análise com 302 passos, índice de consistência 0,55 e índice de retenção 0,87. 
Tabela I. Lista dos gêneros de Cercopidae neotropicais utilizados nas análises.

\begin{tabular}{lll}
\hline Gêneros & Gêneros & Gêneros \\
\hline Gen. nov. 1 & Isozulia & Pachypterinella \\
Aeneolamia & Gen. nov. 8 & Gen. nov. 13 \\
Aracamunia & Kanaima & Gen. nov. 14 \\
Gen. nov. 2 & Gen. nov. 9 & Plagiophleboptena \\
Gen. nov. 3 & Korobona & Prosapia \\
Baetkia & Laccogrypota & Gen. nov. 15 \\
Bradypteroscarta & Gen. nov. 10 & Schistogonia \\
Carpentiera & Mahanarva & Simorhina \\
Catrimania & Makonaima & Sinopia \\
Carachata & Maxantonia & Gen. nov. 16 \\
Choconta & Monecphora & Sphenoclypeana \\
Deois & Neaenus & Sphenorhina \\
Gen. nov. 4 & Neolaccogrypota & Tapaiuna \\
Gen. nov. 5 & Neomonecphora & Tomaspis \\
Hemiplagiophleboptena & Neosphenorhina & Tomaspisina \\
Hemitomaspis & Gen. nov. 11 & Tomaspisinella \\
Gen. nov. 6 & Notozulia & Tropidorhinella \\
Huaina & Gen. nov. 12 & Tunaima \\
Hyboscarta & Ocoaxo & Typeschata \\
Iphirhina & Olcotomaspis & Vorago \\
Gen. nov. 7 & Orodamnis & Zuata \\
Ischnorhina & Pachacanthocnemis & Zulia \\
\hline & &
\end{tabular}

\section{RESULTADOS E DISCUSSÃO}

Na primeira análise, utilizando-se o comando mh*, seguindo-se bb* e com atribuição de pesos sucessivos pelo comando x sw, resultaram 32 cladogramas.

Após, aplicando-se o consenso de Nelson pelo comando ne, resultou um cladograma com 302 passos, índice de consistência 0,55 e índice de retenção 0,87 (Fig. 2). Foram detectadas nove áreas de endemismo na Região Neotropical.

As áreas de endemismo que resultaram agrupadas na primeira análise foram nomeadas seguindo um critério geral e ecológico de caracterização de biomas da Região Neotropical e, também, pela posição geográfica em que se encontram, cujos nomes, não necessariamente, refletem a composição florística. Nomearam-se da seguinte maneira: Área 1-México + América Central: grande parte do México e região norte da América Central; Área 2 - Savana Venezuelana: Venezuela + parte da Colômbia; Área 3-Guiana + Suriname: região oriental da Guiana e ocidental do Suriname; Área 4 - Chaco; Área 5 - Transandina: norte da Cordilheira dos Andes, zona ocidental da Planície Amazônica e sul da América Central; Área 6 - Cerrado; Área 7 - Amazônia; Área 8 - Pampa; e, Área 9-Mata Atlântica (Fig. 3).

Alguns gêneros incluem espécies exclusivas das áreas de endemismo nomeadas (Prosapia Fennah, 1949 - México + América Central; Hemiplagiophleboptena Lallemand, 1949 Chaco; Tomaspisina Distant, 1909 - Trans-andina; Gêneros novos 6 e 13 - Cerrado; e, Pachipterinella Lallemand, 1927 Pampa) justificando-as como áreas válidas de endemismo.

Na segunda análise aplicaram-se os comandos ie (enumeração implícita) e x sw (pesos sucessivos) resultando um único cladograma com 192 passos, índice de consistência 0,80 e índice de retenção 0,85, representando a relação entre as áreas de endemismo anteriormente determinadas (Fig. 4).

Segundo Amorim \& Pires, 1996, vários autores, usando diferentes grupos, indicam pelo menos quarenta áreas de endemismo nucleares na região Neotropical, onde algumas (México + América Central semelhante à área 2; Pampa semelhante à área 48; Mata Atlântica semelhante às áreas 44, 45, 46 e 47; Guiana + Suriname semelhante à área 23; Chaco semelhante à área 35) concordam com as áreas estabelecidas para Cercopidae. Goldani et al. (2002) usando dados de Membracidae, corroboram as áreas México + América Central, e Amazônia.

Analisando o cladograma resultante, nota-se que a área Guiana + Suriname sugere um isolamento mais antigo em relação às demais áreas de endemismo, corroborando resultados semelhantes com vertebrados (SILvA \& OREN 1996). As espécies de Mahanarva Distant, 1909 estão ausentes nesta área.

Carpentiera Lallemand, 1954, Isozulia Fennah, 1953 e Plagiophleboptena Schmidt, 1910 justificam o agrupamento das áreas Chaco e Trans-andina. Gen. nov. 5 e Gen. nov. 10 estão ausentes na região norte da Neotropical. Typeschata Schmidt, 1920 além dos Gêneros novos 4 e 7, agrupam as áreas Pampa e Mata Atlântica, ambas regiões compostas, em parte, por bosques subtropicais decíduos e mesofíticos.

A ocorrência das espécies de alguns gêneros que

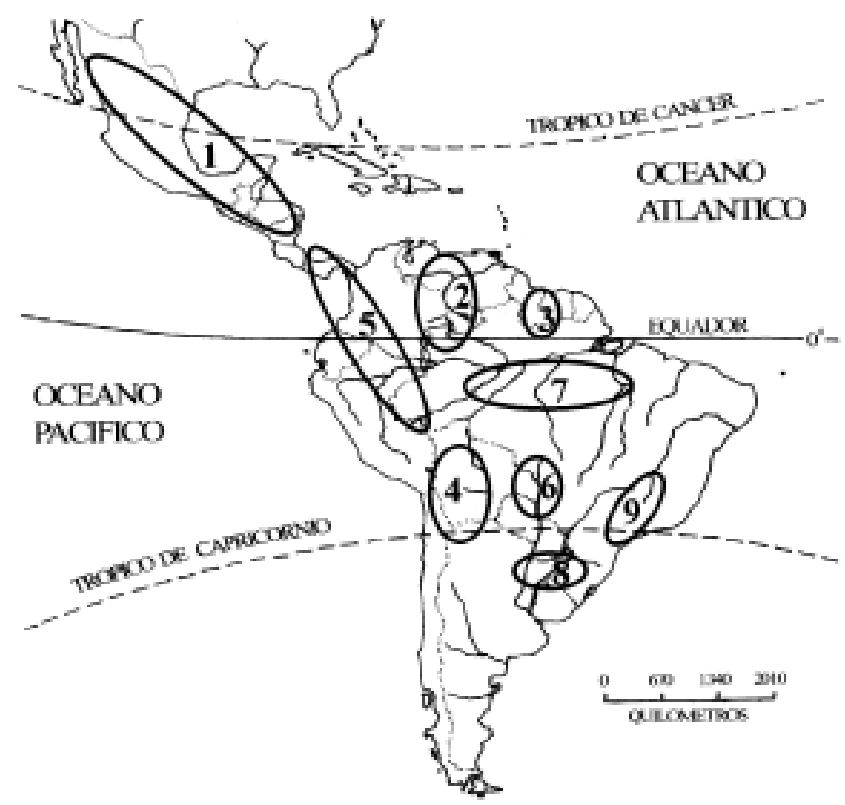

Fig. 3. Mapa da Região Neotropical com as áreas de endemismo estabelecidas. Áreas: 1 - México + América Central: grande parte do México e região norte da América Central; 2 - Savana Venezuelana; 3 Guiana + Suriname: região oriental da Guiana e ocidental do Suriname; 4 - Chaco; 5 - Trans-andina: norte da Cordilheira dos Andes, zona ocidental da Planície Amazônica e sul da América Central; 6 - Cerrado; 7 Amazônia; 8 - Pampa e 9 - Mata Atlântica. 
Tabela II. Matriz de dados da $1^{\text {a }}$ análise com a distribuição dos 66 gêneros de Cercopidae (colunas) nas 49 quadrículas na Região Neotropical (linhas).

$\begin{array}{llllll}1 & 2 & 3 & 4 & 5 & 6\end{array}$

\section{6}

\begin{tabular}{|c|c|}
\hline $\mathrm{GE}$ & 000000000000000000000000000000000000000000000000000000000000000000 \\
\hline Q1 & 010000000000000001000000000000000100010010000000100000000100000000 \\
\hline Q2 & 010000000000000001010000000000000100010011000000100000010100000000 \\
\hline Q3 & 010000000000000001010001000101011010010111000000100000011100000000 \\
\hline Q4 & 010010000010000001000001000101010000110110010000100000011110010001 \\
\hline Q5 & 010010000010000001000000000100000000110010010000000000001110010001 \\
\hline Q6 & 010010000010000000000000000000000000100000100000001000011100000000 \\
\hline Q7 & P 00010000010000000000000000001000000100000100000001000010100000000 \\
\hline Q8 & 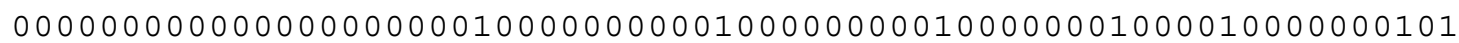 \\
\hline Q9 & 010010100010000001000010000100111000110010010001000001011111110111 \\
\hline Q10 & 010110000010000001000010000100000010110010010000000000001110010011 \\
\hline Q11 & 000010000010000000000000000000000000100000100000001000011100000000 \\
\hline Q12 & P 10010000010000000000000000000000000100000000000001000010100000000 \\
\hline Q13 & 010000000000000000000100000000011000000000100000001000010000000101 \\
\hline Q14 & 000100100001000000100110000101111010000010010001010101011111000101 \\
\hline Q15 & 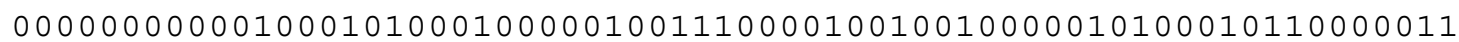 \\
\hline Q16 & 100000000001010100000000000111000000000000100000010000000000000111 \\
\hline Q17 & 100000001001010000000000000011000000000000100000011000000000000111 \\
\hline Q18 & 110001000001010000000100010111010000000010100000010000000000000111 \\
\hline Q19 & 110000000001010000000100010111000000000010100000010000000000000001 \\
\hline Q2 0 & 000000000000000100000110000100111000000000000000010100010110000011 \\
\hline Q21 & 000000000001010100000100000110111000000000000000010100010110000011 \\
\hline Q22 2 & 100000000001010000000000000011000000000000100000010100000000000111 \\
\hline Q23 & 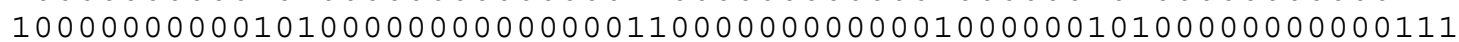 \\
\hline Q24 & 110001000001010000000100010111000000000010100000010000000000000111 \\
\hline Q25 & 110000001001010000000100010111000000000010100000010000000000000001 \\
\hline Q26 & p \\
\hline Q2 7 & 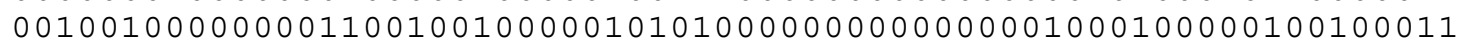 \\
\hline Q2 8 & 111001000100011100100100000111010000000000000001000100000100100011 \\
\hline Q2 9 & 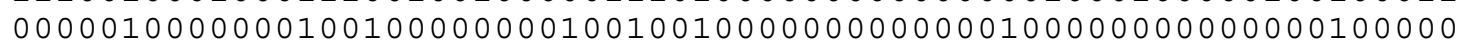 \\
\hline Q30 & 000001000001010010000000010010010000000000000100000000000000100000 \\
\hline Q31 & 000000000000010000000100001111010000100000000000011000110100010000 \\
\hline Q32 & 000000000000000100000100000000011000000000000000000100010110000000 \\
\hline Q33 & 001001010000001100100110000101010000000000000001000100000100100011 \\
\hline Q34 & 001001000000001100100100000101010000000000000001000100000100100011 \\
\hline Q35 & 100001000001010010000000010011000000000000000100000000000000100000 \\
\hline Q36 & 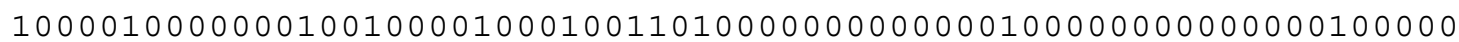 \\
\hline Q37 & 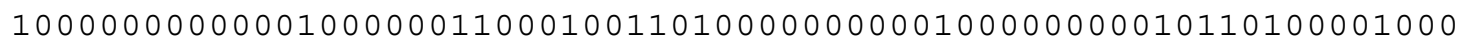 \\
\hline Q38 & 100000000000010000001100000011010001100000100010000010110100001000 \\
\hline Q39 & 000000000000001100100110000001010000000000100011000000000100000011 \\
\hline Q4 0 & 000100000000001100100110000001010000001000100011000000100100000011 \\
\hline Q4 1 & 100100000000010000000000000011000000001000000000000000100100000010 \\
\hline Q4 2 & 110000000001110000000100011011000000101000000000000000110100000000 \\
\hline Q4 3 & 100000000001110000001100011011010000100000100010000010110100010000 \\
\hline Q44 4 & 100000000101010001001100000011011001100000100010000010110100011000 \\
\hline Q4 5 & 100100000001010000000000000011000000001000101010000000100100000010 \\
\hline Q46 & 110000000001110000101000000011010000101000001010000000100100011000 \\
\hline Q4 7 & 100000000000010000101000100010010000100000001000000000100000011010 \\
\hline Q48 & 100000000001010000001000000011000000000000101010000000100100000000 \\
\hline Q4 9 & 100000000001110000000000100011000000000000001010000000100100010000 \\
\hline
\end{tabular}

Gêneros: 1-Gen. nov. 1, 2-Aeneolamia, 3-Aracamunia, 4-Gen. nov. 2, 5-Gen. nov. 3, 6-Baetkia, 7-Bradypteroscarta, 8-Carpentiera, 9-Catrimania, 10-Carachata, 11-Choconta, 12-Deois, 13-Gen. nov. 4, 14-Gen. nov. 5, 15-Hemiplagiophleboptena, 16-Hemitomaspis, 17-Gen. nov. 6, 18-Huaina, 19-Hyboscarta, 20-Iphirhina, 21-Gen. nov. 7, 22-Ischnorhina, 23-Isozulia, 24-Gen. nov. 8, 25-Kanaima, 26-Gen. nov. 9, 27-Korobona, 28-Laccogrypota, 29-Gen. nov. 10, 30-Mahanarva, 31-Makonaima, 32-Maxantonia, 33-Monecphora, 34-Neaenus, 35-Neolaccogrypota, 36-Neomonecphora, 37-Neosphenorhina, 38-Gen. nov. 11, 39-Notozulia, 40-Gen. nov. 12, 41-Ocoaxo, 42-Olcotomaspis, 43-Orodamnis, 44Pachacanthocnemis, 45-Pachypterinella, 46-Gen.nov.13, 47-Gen. nov. 14, 48-Plagiophleboptena, 49-Prosapia, 50-Gen. nov. 15, 51-Schistogonia, 52-Simorhina, 53-Sinopia, 54-Gen. nov. 16, 55-Sphenoclypeana, 56-Sphenorhina, 57-Tapaiuna, 58-Tomaspis, 59-Tomaspisina, 60-Tomaspisinella, 61-Tropidorhinella, 62-Tunaima, 63-Typeschata, 64Vorago, 65-Zuata, 66-Zulia. 
Tabela III. Matriz de dados da $2^{\mathrm{a}}$ análise com a distribuição dos 48 gêneros de Cercopidae (colunas) nas áreas de endemismo determinadas na $1^{\mathrm{a}}$ análise (linhas) na Região Neotropical.

\section{4}

123456789012345678901234567890123456789012345678

G E

México + América Central

Savana Venezuelana

Chaco

Trans-andina

Cerrado

Amazônia

Pampa

Mata Atlântica

Guiana + Suriname
000000000000000000000000000000000000000000000000

010000000000100001010101110101100010000111000000

111011100011010110011101000010011100011001100011

011101011011110111011111111101100111010111110111

100010001010000100101101000000000000000000100000

110010001011000100111101000000110001110000000111

111000001110011000001101001010011000001001011010

110000101110101100111101101010011001101101011000

010000000000000100000001100000010000100100000101
010100010000000000000100001000010000100111000000

Gêneros: 1-Gen. nov. 1, 2-Aeneolamia, 3-Gen. nov. 2, 4-Gen. nov. 3, 5-Baetkia, 6-Carpentiera, 7-Carachata, 8-Choconta, 9-Deois, 10-Gen. nov. 4, 11-Gen. nov. 5, 12-Hemitomaspis, 13-Huaina, 14-Hyboscarta, 15-Gen. nov. 7, 16-Ischnorhina, 17-Isozulia, 18-Gen. nov. 8, 19-Gen. nov. 9, 20-Laccogrypota, 21-Gen. nov. 10, 22-Mahanarva, 23-Makonaima, 24-Maxantonia, 25-Monecphora, 26-Neolaccogrypota, 27-Neosphenorhina, 28-Gen. nov. 11, 29-Notozulia, 30-Gen. nov. 12, 31-Ocoaxo, 32-Orodamnis, 33-Gen. nov. 14, 34Plagiophleboptena, 35-Prosapia, 36-Gen. nov. 15, 37-Schistogonia, 38-Simorhina, 39-Sphenoclypeana, 40-Sphenorhina, 41-Tapaiuna, 42-Tomaspis, 43-Tropidorhinella, 44Tunaima, 45-Typeschata, 46-Vorago, 47-Zuata, 48-Zulia.

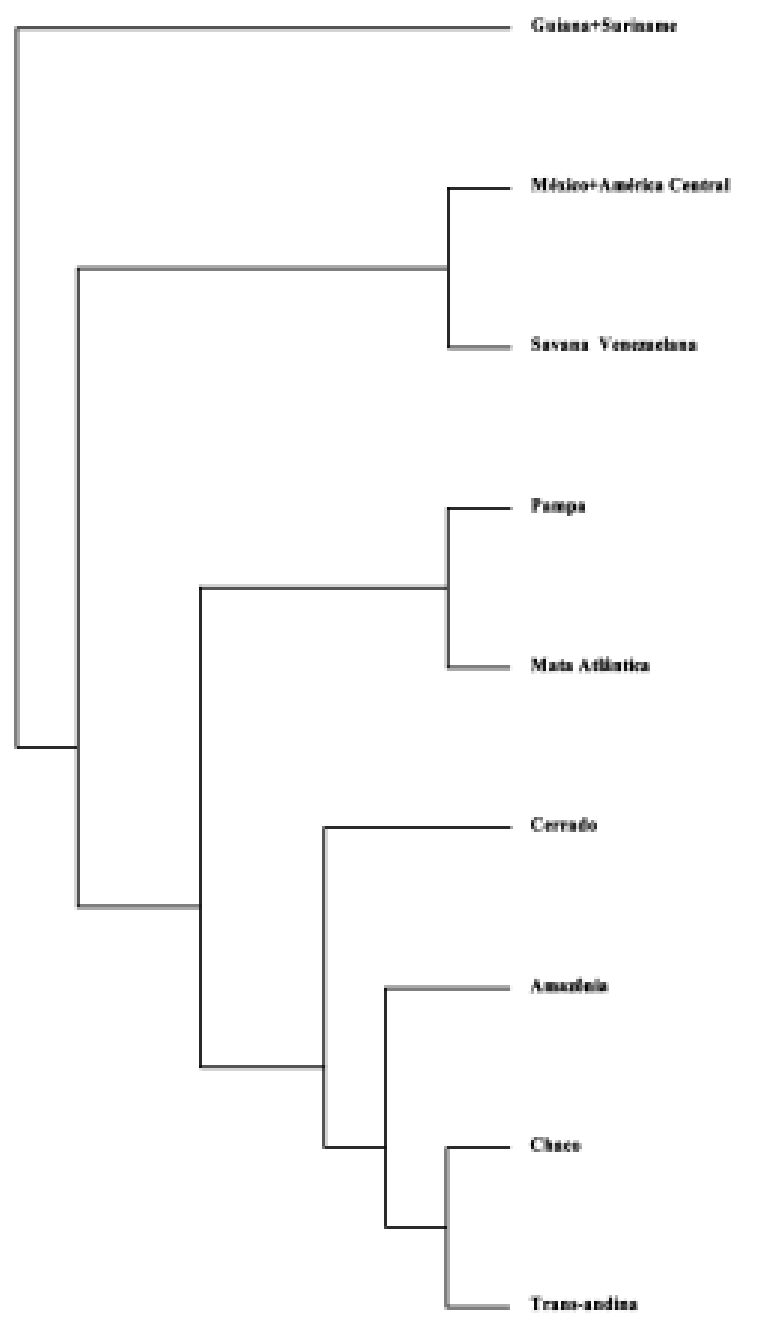

Fig. 4. Cladograma resultante da $2^{\mathrm{a}}$ análise com 192 passos, índice de consistência 0,80 e índice de retenção 0,85 . possibilitaram agrupamentos de áreas, em ambas análises, refletem uma composição ecológica mista das mesmas, indicando a preferência alimentar desses insetos.

Algumas ausências nas matrizes podem ser explicadas pela falta de coleta, justificando a importância de dados adicionais em estudos futuros, além da utilização de outros grupos taxonômicos para uma maior compreensão das áreas de endemismo da Região Neotropical.

Agradecimentos. Aos consultores anônimos, pelas sugestões valiosas e revisão do manuscrito. Ao CNPq pelo auxílio financeiro.

\section{REFERÊNCIAS}

Amorim, D. S. \& M. R. S. Pires. 1996. Neotropical Biogeography and a Method for Maximum Biodiversity Estimation, p. 183-219. In: C. E. M. Bicudo \& N. A. Menezes (eds.) Biodiversity in Brazil: a first approach. São Paulo, CNPq.

CRisci, J. V.; L. Katinas \& P. PoSADAs. 2000. Introduccion a la teoria y practica de la biogeografia historica. Buenos Aires, Sociedad Argentina de Botánica, 169 p.

Espinosa-Organista, D. \& J. Llorente-Bouquets. 1993. Fundamentos de Biogeografías Filogenéticas. México, Universidad Nacional Autónoma de México, 133 p.

FARRIS, J. S. 1988. Hennig86 reference. Version 1.5. Published by the author, New York, Port Jefferson Station.

Goldani, Â; A. Ferrari; G. S. Carvalho \& A. J. CreÃo-Duarte. 2002. Análise de parcimônia de endemismo de membracídeos neotropicais (Hemiptera, Membracidae, Hoplophorionini). Revista Brasileira de Zoologia 19 (Supl.2): 187-193.

Harold, A. S. \& R. D. Moor. 1994. Areas of Endemism: Definition and Recognition Criteria. Systematic Biology 43(2): 261-266.

Metcalf, Z. P. 1961. General Catalogue of the Homoptera. Fasc. VII, Part 2, Cercopidae. Raleigh, N. C. North Carolina State College, $616 \mathrm{p}$.

Morrone, J. J. 1994. On the identification of areas of endemism. Systematic Biology 43(3): 438-441. 
Myers, A. A. 1991. How did Hawaii accumulate its biota? A test from the Amphipoda. Global Ecological Biogeography Letts 1: 24-29.

Platnick, N. I. \& G. Nelson. 1978. A method of analysis for historical biogeography. Systematic Zoology 27(1): 1-16.

Rosen, B. R. 1988. From fossils to earth history: applied historical biogeography, p. 437-481. In: A. A. Myers \& P. S. Gillers (eds.). Analytical Biogeography. London, Chapman \& Hall, 578 p.

Rosen, B. R. \& A. B. Sмiтн. 1988. Tectonics from fossils? Analysis of reef-coral and sea-urchin distribution from late Cretaceous to Recent, using a new method. Gondwana and Tethys, p. 275-305. In: M. G.
Audley-Charles \& A. Hallam (eds.). Geological Society Special Publication 37. Oxford, Clarendon Press, 317 p.

Silva, J. M. C. \& D. C. Oren. 1996. Application of parsimony analysis of endemicity in Amazonian biogeography: an exemple with primates. Biological Journal of the Linnean Society 59: 427-437.

Zunino, M. 2000. El concepto de Área de Distribución: algunas reflexiones teóricas, p. 79-85. In: F. Martín-Piera, J. J. Morrone \& A. Melic (eds.). Hacia um Proyecto CYTED para el Inventario de la Diversidad Entomológica en Iberoamérica: Pribes 2000. Zaragoza, Sociedad Entomológica Aragonesa, 326 p. 